\title{
AngioVac Suction Embolectomy Using a Roller Pump: Operative Technique
}

\author{
Michael Shang ${ }^{1}$, Touraj Rajabipour ${ }^{1}$, and Prashanth Vallabhajosyula ${ }^{1}$ \\ ${ }^{1}$ Yale School of Medicine
}

June 13, 2021

\begin{abstract}
AngioVac suction embolectomy is recommended by the manufacturer to be performed with a centrifugal pump due to safety considerations. However, roller head pumps are significantly cheaper to use, and thus may be more readily available during shortages and in resource poor settings. We present the technique of Angiovac suction embolectomy being successfully performed with a roller pump to evacuate a clot-in-transit in the inferior vena cava and right atrium, along with discussion of important safety caveats.
\end{abstract}

\section{AngioVac Suction Embolectomy Using a Roller Pump: Operative Technique}

Running title: Roller pump suction embolectomy

Michael Shang BS${ }^{1}$, Touraj Rajabipour BS, MHS, CCP${ }^{1}$, Prashanth Vallabhajosyula MD, MS

Division of Cardiac Surgery, Yale University School of Medicine, New Haven, CT

Conflict of Interest: We have no conflicts of interest to report.

Sources of Funding: We have no sources of funding to report.

Institutional Review Board: Institutional Review Board review was waived under guidelines set forth by our institution.

Informed Consent: Informed consent was waived as the subject of the report is deceased and the information presented is de-identified according to safe-harbor deidentification guidelines. To our knowledge, the information presented cannot be used alone or in combination with other information to uniquely identify the subject.

\section{Corresponding Author:}

Prashanth Vallabhajosyula

330 Cedar St. PO Box 208062

New Haven, CT 06520-8062

Email: prashanth.vallabhajosyula@yale.edu

Phone: 203-785-5000

Word count: 1408

Abstract 
AngioVac suction embolectomy is recommended by the manufacturer to be performed with a centrifugal pump due to safety considerations. However, roller head pumps are significantly cheaper to use, and thus may be more readily available during shortages and in resource poor settings. We present the technique of Angiovac suction embolectomy being successfully performed with a roller pump to evacuate a clot-in-transit in the inferior vena cava and right atrium, along with discussion of important safety caveats.

Text

\section{Introduction}

The AngioVac device (AngioDynamics, Latham, NY) is currently approved for suction embolectomy of venous thromboemboli. The AngioVac system consists of a catheter with a funnel-shaped tip connected to a veno-venous (VV) extracorporeal bypass circuit that filters aspirated blood before returning it systemically. The literature documenting its use unanimously reports using a centrifugal pump, as recommended by the manufacturer. ${ }^{1-3}$ However, roller pumps are an attractive alternative in this situation due to lower cost, especially in resource poor settings or during times of severe equipment shortage. Such a situation occurred at our institution during the peak of the COVID-19 pandemic. We report the use of a roller pump with the AngioVac device to extract a right atrial (RA) clot-in-transit in a patient with pulmonary embolism (PE) and severe cardiogenic shock.

Informed consent was waived under guidelines set forth by our institution's Institutional Review Board.

\section{Case}

A 76-year-old male with history of coronary artery disease and chronic obstructive pulmonary disease presented to an outside hospital with acute-onset dyspnea. The patient was hemodynamically stable with initial laboratory evaluation significant for anion-gap metabolic acidosis, venous lactate 7.6, D-dimer 11.65, and proBNP 17,056; SARS-CoV-2 RNA testing was negative. CT angiography of the chest revealed saddle pulmonary embolus with near complete occlusion of the main pulmonary arteries and evidence of right ventricle (RV) strain. The patient was treated initially with intravenous heparin. Despite this, the patient began experiencing increasing dyspnea with repeat echocardiogram showing a new $4.8 \mathrm{~cm}$-by- $1.4 \mathrm{~cm}$ mobile thrombus in the RA going in and out of the RV during the cardiac cycle with additional clot extending into the inferior vena cava (IVC) (Figure 1 ). Due to concerns for distal embolization causing massive PE, thrombolytic therapy was deferred and the patient was transferred to our facility for urgent suction embolectomy using the AngioVac system.

\section{Operative Technique}

The procedure was performed using C-arm fluoroscopy and transesophageal echocardiography (TEE) by a cardiac surgeon experienced in catheter-based and surgical embolectomy. Intraoperative TEE demonstrated a markedly dilated RV and underfilled left ventricle (LV) with hyperdynamic LV function. Upon general anesthesia induction and intubation, guidewires were placed in both common femoral veins (CFV) and advanced into the IVC under fluoroscopic guidance. The patient was anticoagulated with heparin, and next, a 26F dry-seal sheath (Gore Medical) was placed in the right CFV for the AngioVac cannula and an $18 \mathrm{~F}$ cannula was placed in the left CFV for the reinfusion cannula. The AngioVac catheter was introduced through the right groin sheath and advanced into the IVC prior to connecting it to the venous inflow tubing of the VV bypass circuit after proper deairing maneuvers. The system was then connected to the roller pump. The AngioVac catheter was carefully advanced under fluoroscopic and echocardiographic guidance, as the pump was started at $1 \mathrm{~L} / \mathrm{min}$ and gradually increased to flow rate of $2.5 \mathrm{~L} / \mathrm{min}$ under fluoroscopic and echocardiography guidance. The catheter was then advanced to the IVC-RA junction, at which point a large thrombus was suctioned into the filter (Figure 2 ). A second thrombus was evacuated upon further advancement into the RA. We verified that no remaining clot was visible in the RA and RV using TEE and proceeded to come off VV bypass by shutting down the pump gradually. Both cannulas were removed, and groin sites repaired. The entire procedure was without complications.

\section{Discussion}


The AngioVac system is recommended for use with centrifugal pumps because they are non-occlusive with no risk for cavitation of air. However, the present case was performed at the height of the COVID-19 pandemic, during which our hospital was experiencing a severe shortage in centrifugal pump heads due to the sudden increase in utilization for ECMO support in COVID patients. Given the urgent status of the procedure, we decided to use a roller pump as it was the only equipment available at the time. In addition, roller pumps have the added benefit of being significantly cheaper to use as they do not require replacement of a centrifugal pump head, which costs $\$ 12,600$ per use.

Though the present case was technically successful and without complications, there are important safety considerations requiring careful evaluation with the use of roller pumps. First, roller pumps are occlusive and will continue to pump against any resistance, making them more likely to result in significant structural damage if the AngioVac catheter tip gets suctioned against an anatomic structure (i.e. inferior vena cava, right atrial free wall). Therefore, very gradual flow rate changes, capping at a low flow rate $(2.5 \mathrm{~L} / \mathrm{minute}$ in our case), continuous visualization of the device and short advancements, with the operative team ready to make immediate adjustments, are required. Additionally, the AngioVac catheter has been reported to clog when used to evacuate solid or chronic thrombi. ${ }^{2}$ With a roller pump, a fully occluded tip may result in cavitation of air as well as trauma to blood elements due to shear stress from being suctioned around the occlusion. ${ }^{4}$ These considerations are especially important as introduction of air into the circuit can potentially be catastrophic in patients with PE who may already have RV dysfunction. While roller pumps may be more cost effective, safety is paramount. Thus, we recommend that this technique only be considered for soft, fresh thrombi in locations where navigation of the suction tip around delicate structures and long advancements of the device are not required.

In the present case, we demonstrate that AngioVac suction embolectomy can be safely and effectively performed with a roller pump to evacuate mobile, acute thrombi in the IVC and RA. In situations where centrifugal pumps are inaccessible, roller head pumps may be considered as an alternative under specific circumstances, granted precautions are taken to adjust for its limitations.

\section{Author Contributions}

MS contributed to the concept and drafting of the manuscript.

TR obtained cost data and contributed to the critical revision and approval of the manuscript.

PV performed the procedure reported in the article and contributed to the concept, critical revision, and approval of the manuscript.

\section{References}

1. Resnick, S.A., D. O'Brien, D. Strain, C. Malaisrie, D. Schimmel, R. Salem, et al. Single-Center Experience Using AngioVac with Extracorporeal Bypass for Mechanical Thrombectomy of Atrial and Central Vein Thrombi. J Vasc Interv Radiol . 2016;27:723-729 e1.

2. Schultz, J., A. Andersen, E.L. Grove, L.B. Ilkjaer, and J.E. Nielsen-Kudsk. Large Solid Right Atrial Thrombus Treated by AngioVac Catheter-Based Suction Thrombectomy. Case Rep Cardiol . 2018;2018:7904064.

3. Michelson, C.M., C.M. Dyke, D.J. Wick, R. Guenther, D. Dangerfield, and M.E. Wiisanen. Use of a Modified Cardiopulmonary Bypass Circuit for Suction Embolectomy with the AngioVac Device. J Extra Corpor Technol . 2017;49:299-303.

4. Datt, B., M.B. Nguyen, G. Plancher, M. Ruzmetov, M. O'Brien, A. Kube, et al. The Impact of Roller Pump vs. Centrifugal Pump on Homologous Blood Transfusion in Pediatric Cardiac Surgery. J Extra Corpor Technol . 2017;49:36-43.

Figure Legends 
Figure 1: Transthoracic echocardiogram showing a $4.8 \mathrm{~cm} \times 1.4 \mathrm{~cm}$ thrombus in the right atrium and extending into the inferior vena cava.

RA: right atrium

TV: tricuspid valve

IVC: inferior vena cava

T: thrombus

Figure 2: Evacuated thrombus from inferior vena cava and right atrium shown in the AngioVac filter system.

\section{Figures}

\section{Figure 1:}

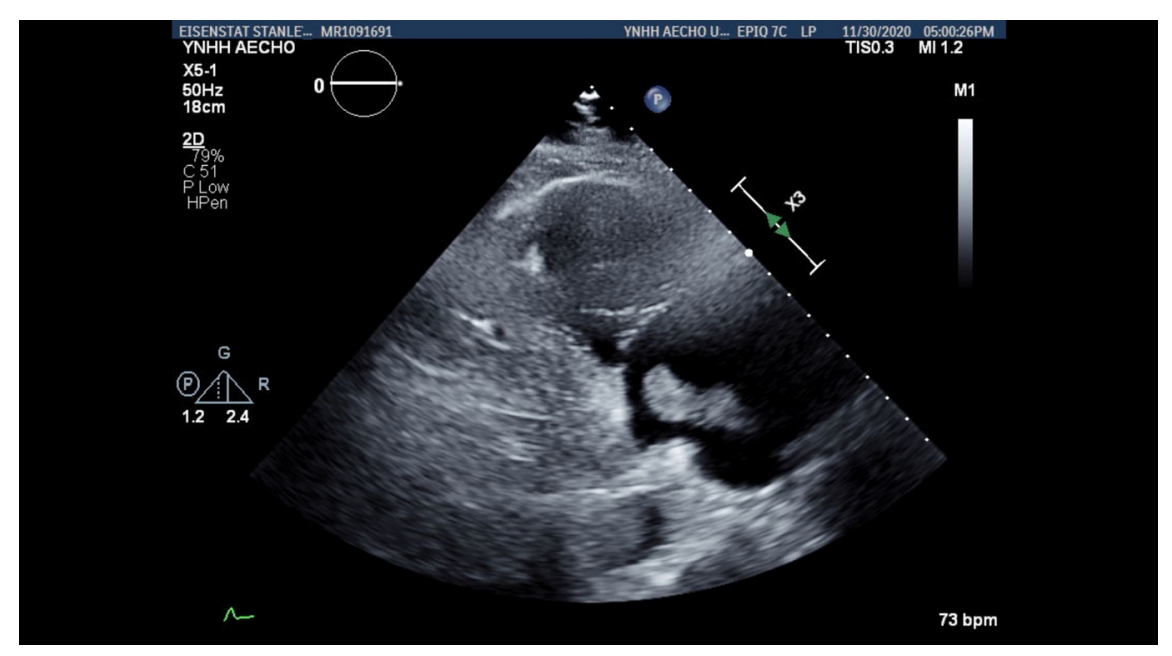

Figure 2: 


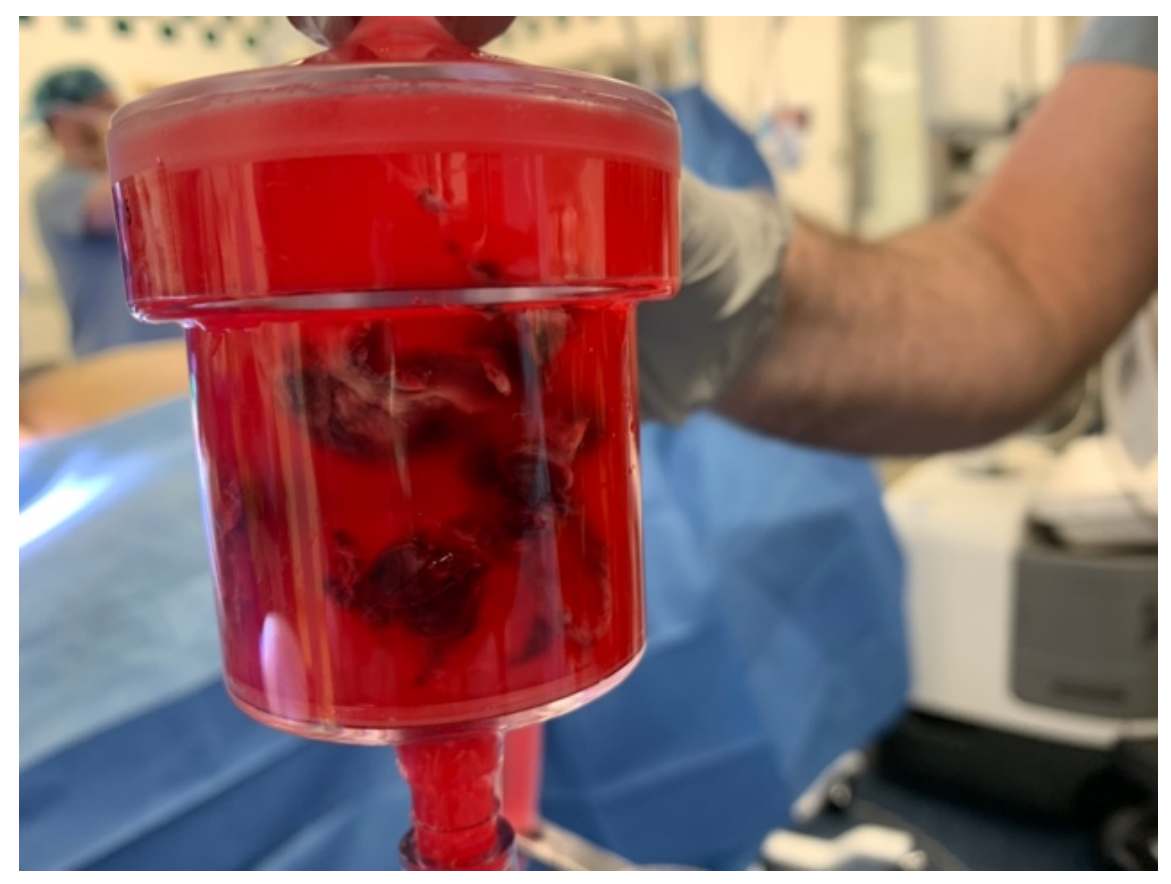

